Archivo del CID recibe

Fondo Emilio Duhart

Durante el mes de abril pasado Emilio Duhar (arquitecto PUC, 1941, Premio Nacional de Arquitectura 1977) hizo una importante donación al Centro de Información y Documentación Sergio Larraín GarcíaMoreno (CID- SLGM). Duhart, quien actualmente reside en París, entregó a CID gran parte de su archivo de trabajo, que incluye aproximadamente 2.800 planos entre originales y copias de 50 proyecto desarrollados en su dilatada labor profesional, tanto en Chile como en Francia. Una vez catalogado, este conjunto de documentos quedará a disposición de investigadores y estudiantes, cumpliendo con uno de los objetivos fundamentales que se ha propuesto la Facultad a través del CID: prestar un servicio al desarrollo cultural del país y al mismo tiempo, convertirse en un importante archivo patrimonial de arquitectura, abierto a investioadores en el ámbito nacional e internacional, recopilando, preservando $y$ difundiendo el patrimonio arquitectónico y urbano de Chile.

tinfo: Pedro Ignacio Alonso, coordinador Archivo de

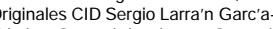
cid-slgm@ puc.cl / palonsoz@ puc.cl, tel. (56 2)686-5584 /

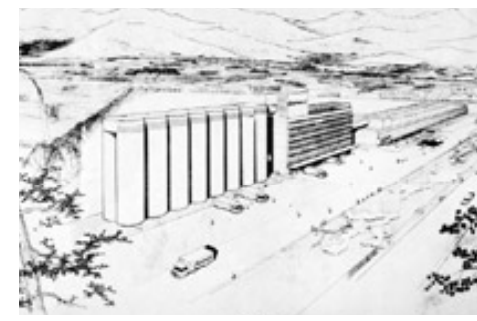

(Cojo Industrial Carozzi (Construido), 1961 Perspectiva.

CID online

Fue aprobado el proyecto de desarrollo de la docencia FONDEDOC 2002 presentado por el ex- decano de la Facultad Fernando Pérez y el arquitecto Claudio Labarca, orientado a la implementación de la página web del Centro de Información y Documentación Sergio Larraín G-M. La iniciativa permitira acceder a los archivos del CID a través de Internet, lo que facilitará el uso y estudio de los documentos que aquí se conservan; se espera que en el mediano plazo un porcentaje importante de los archivos estén digitalizados y catalogados en este nuevo formato de consulta.

Rafael Islesia en Chile

Extensión FADEU y Procobre inician hacia fines de agosto un programa de visitas de arquitectos latinoamericanos a nuestra escuela. Se trata de abrir una conversación respecto al desarrollo de cierta sensibilidad hacia los materiales como problema de arquitectura, con profesionales que comparten con nosotros realidades tecnológicas, sociales y geográficas. El primer invitado de esta iniciativa será el argentino Rafael Iglesia (parte de su obra se publica en esta edición de ARQ); Iglesia participará como profeso en un taller en el ámbito del Magister de Arquitectura de la Facultad y dará una charl abierta a estudiantes de arquitectura.
Alejandro Morales premiado en Concurso Internacional

Alejandro Morales (arquitecto PUC, 1996) recibió uno de los veinte premios especiales en el concurso Northern Style Housing Complex, jurado en febrero de este año en Japón. El jurado, presidido por Jean Nouvel y Tadao Ando, seleccionó 5 finalistas y 20 premios especiales entre 970 trabajos, llegados de todo el mundo.

El concurso convocaba a la presentación de proyectos para un edificio multifuncional de $40.000 \mathrm{~m} 2$ que incluía viviendas, comercio, teatro y equipamiento en el contexto del centro urbano de la ciudad de Aomori (cubierta de nieve tres meses al año). La iniciativa proponía la densificación del casco antiguo de la ciudad para evitar la expansión de los suburbios; uno de los problemas que el proyecto planteaba era cómo cualificar las condiciones de vida de un esquema de alta densidad de manera de hacerlo competitivo con la oferta de viviendas "espaciosas" y ajardinadas de las nuevas periferias.

\section{Premiación de Aulas de Titulación}

Escuela de Arquitectura PUC

En abril del presente año se realizó la entrega de premios para Aulas de Titulación. En ésta se premió como Aula con mejor promedio la de Alejandro Gutiérrez y Luis Valenzuela, cuyo tema era Intervenciones en el Gran Valparaíso. Como proyectos mejor evaluados fueron galardonados los de Atilio Caorsi y el de Braulio Morera, ambos del aula GutiérrezValenzuela.

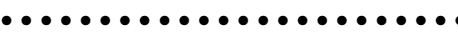

Arquitectos chilenos en

\section{Santo Domingo}

El Ayuntamiento de la Ciudad de Santo Domingo convocó a 27 equipos profesionales de todo el mundo -entre los que destacan Manuel Solá Morales, Henk Döl (Mecanoo) y Clorindo Testa- para elaborar propuestas urbanas para esa ciudad como parte de un plan de intervenciones de recuperación. Entre los invitados, se encuentran los arquitectos chilenos Humberto Eliash, Mario Torres y Sebastián Irarrázaval. El proyecto de éste último -una intervención en el área del Parque Enriquillo, que contempla la renovación del espacio público en calles y la construcción de una plaza techada- fue destacado por las autoridades dominicanas por su escala e impacto en la ciudad; actualmente se evalúa la posibilidad de construirlo en el corto plazo.

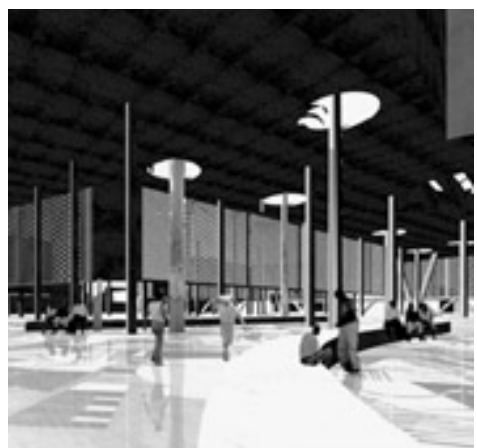

Exposición de Arquitectura Chilena en Barcelona

Durante el mayo y junio se pudo apreciar en la sede del Colegio de Arquitectos de Cataluña, en Barcelona, la exposición "Condiciones de Lejanía: Espacio Contemporáneo en Chile", ideada y curada por Pilar Calderón y Marc Folch. En ésta se mostraba la obra de 15 arquitectos chilenos, entre los cuales se encontraban Alejandro Aravena, Sebastián Irarrázaval, Cazú Zegers y José Cruz, los cuales participaron a su vez en el ciclo de charlas asociado a la exposición. Al mismo tiempo, y relacionada a la exposición anterior, se encontraban otras dos muestras; una relacionada a la Escuela de Arquitectura de la UCV y la otra al Taller de Juan Borchers.

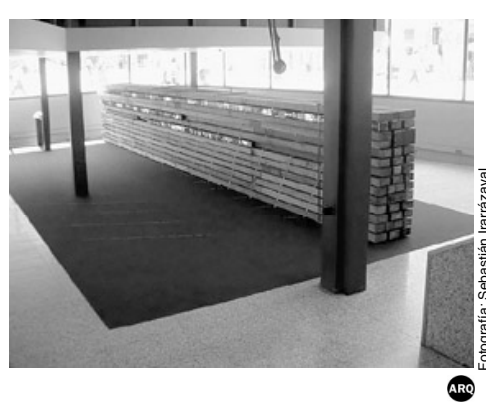

Concurso Bticino

Durante julio, Bticino y Ediciones ARQ lanzarán un concurso para profesionales y estudiantes de arquitectura y diseño. Podrán participar egresados y estudiantes de todas las escuelas del país, individualmente o en equipos dirigidos por un profesor: el encargo del concurso es un proyecto de intervención lumínica para el pabellón de Barcelona de Mies Van der Rohe. Los proyectos deben explorar la idea de evento, presentando digitalmente una secuencia a través de una maqueta virtual y un recorrido por el pabellón. El premio para el equipo ganador es un viaje a Milán para dos personas, incluyendo una visita a una feria internacional y U\$ 1.000 ; el segundo premio es un computador Mac G4, $600 \mathrm{Mhz}, 64 \mathrm{Mb}$ RAM y monitor 17". Habrá dos menciones honrosas que recibirán cuentas de $\$ 150.000$ para compras en librerías; entre todos los participantes se sortearán productos Bticino. Inscripciones: entre el 12 y el 23 de agosto de 2002
Fecha de entrega: 3 de octubre de 2002
tinfo: argedic@ puc.cl

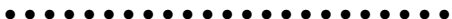

Encuentro de Estudiantes de Arquitectura

Entre los días 21 y 23 de agosto se realizará en el Campus Lo Contador de la PUC un encuentro de Estudiantes de Arquitectura de todo el país, organizado por el Centro de Estudiantes de la Escuela de Arquitectura de la PUC (CEEA). Entre las diversas actividades se contemplan foros, mesas, conferencias de arquitectos chilenos, la visita del arquitecto paraguayo Solano Benítez y la elección del mejor arquitecto chileno joven.

info wwweact o a email ceeapucct

\section{Seminario sobre Zonas áridas}

Durante el mes de noviembre del presente año se realizará en nuestra Escuela el Seminario denominado Diseño Urbano en Zonas Áridas, el cual contará con la organización y participación de la Facultad de Arquitectura, Diseño y Estudios Urbanos de la Pontificia Universidad Católica de Chile en conjunto con la Facultad de Arquitectura de la Universidad de Arizona (Tucson, EEUU).

El seminario plantea una primera aproximación multidisciplinar y comparativa entre las situaciones similares de Santiago y Arizona ahondando en una visión crítica de los problemas de la ciudad, el paisaje y la arquitectura. El diseño del seminario contempla diversas entradas al problema -política, cultural, geográfica, arquitectónica, urbanística y del paisaje-y contará con la visita y participación de Thomas Sheridan, Carol West, Ignacio San Martín, Nader Chalfoun, Christy Ten Eyck, Alvaro Malo y Less Wallach, desde Arizona. +info: fchateau@ puc.cl

Premio Nacional de Arquitectura 2002

En el mes de julio se le concedió el Premio Nacional de Arquitectura 2002 al arquitecto Juan Sabbagh Pisano, el cual le será entregado en el transcurso de la XIII Bienal de Arquitectura.

Sabbagh se ha destacado en el ámbito profesional y en el académico, siendo característico su trabajo en el diseño de arquitectura industrial.

Habiendo difundido su obra en nuestras publicaciones, aprovechamos, como equipo ARQ de felicitarlo a él y a su oficina.

Premio ar+d para arquitectos y diseñadores emergentes

Hasta el 17 de septiembre la revista Architectural Review recibirá trabajos de arquitectura, paisajismo, diseño industrial, interiorismo y diseño urbano para postular al premio ar+d 2002. A este premio, promovido por la revista en conjunto con la empresa $d$ lin international, pueden postular profesionales menores de 45 años, presentando sólo obras construidas: uno de los objetivos es, según sus organizadores, reconocer el paso exitoso de la teoría a la práctica y del dibujo a la construcción. Este año se repartirán $£ 10.000$ entre los ganadores de las diferentes categorías, que se darán a conocer en noviembre en Copenhagen. El premio además incluye la publicación de las obras en la edición de diciembre de Architectural Review y una invitación a Londres para participar en la Temporada de Conferencias del Royal Institute of British Architects en la primavera del 2003.

tinfo: www. arplusd.com / e-mail: lynne.jackson@ebcemap.com

Fe de erratas

Por razones ajenas a nuestra voluntad en el número 50 de nuestra revista se omitió el nombre de Juan José Ugarte, director de la Escuela de Arquitectura de la P.U.C, como miembro del Comité editorial ARQ Lamentamos este error y aseguramos que estamos buscando a los culpables.

Por otro lado, en el artículo "Hoffmann' House", de la página 36, hubo una equivocación en el pie de imagen 2-3; donde dice Cristina Silva debe decir Cristián Silva.

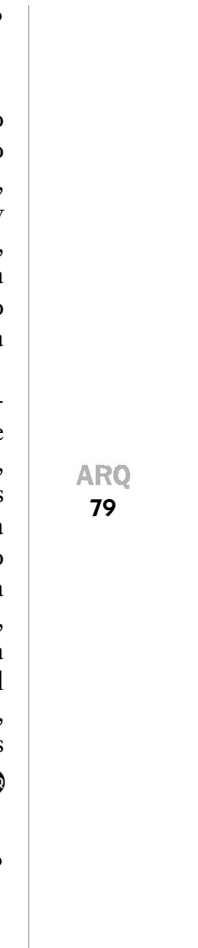
\title{
.
}

